Jolanta Hinc

Uniwersytet Gdański

jolanta.hinc@ug.edu.pl

\title{
ZUR FÖRDERUNG DER INDIVIDUELLEN MEHRSPRACHIGKEIT DES FREMDSPRACHENLERNENDEN - AUFGABEN FÜR DIE POLNISCH- UND DIE FREMDSPRACHENLEHRENDEN
}

\author{
Promoting individual multilingualism of foreign language learner - \\ tasks for the Polish and foreign language teachers
}

This paper relates to the concept of didactics of multilingualism. The didactics of multilingualism is geared towards promoting the language awareness of pupils and showing that knowing more languages makes the learning process more efficient. This can be realized by comparing grammar structures, comparing vocabulary, discussing sociocultural aspects or taking advantage of the intercomprehension. The aspect of the negative transfer should be also captured by showing the interference fields and discussing the interference errors. The didactics of multilingualism should be understood as a continuous process that begins with the acquisition of the mother tongue and goes further over the acquiring the first foreign language and the next foreign language. Following this, the didactics of multilingualism should involve tasks for teachers of the Polish language and teachers of the first, second and next foreign languages.

Keywords: multilingualism, didactics of multilingualism, tasks for teachers of the Polish language and foreign languages

Słowa kluczowe: wielojęzyczność, dydaktyka wielojęzyczności, zadania dla nauczycieli języka polskiego i nauczycieli języków obcych 


\section{Einleitung}

Der folgende Beitrag setzt sich aus zwei Teilen zusammen. Im ersten Abschnitt wird der Begriff der Mehrsprachigkeit definiert und das Konzept der Mehrsprachigkeitsdidaktik, das im schulischen System realisiert werden kann, vorgestellt. Im zweiten Teil wird die Frage diskutiert, wie man die individuelle Mehrsprachigkeit des Lernenden im Fremdsprachenunterricht fördern kann. Diese Aufgabe wird als ein Kontinuum verstanden. Der Prozess der Förderung der Mehrsprachigkeit sollte dementsprechend mit dem Erwerb der Muttersprache im Kindergarten oder im Primarbereich der Grundschule seinen Anfang nehmen und im Laufe der Ausbildung gezielt fortgesetzt werden. Im Anschluss daran werden im vorliegenden Beitrag Aufgaben für die Polnisch- und Fremdsprachenlehrenden präsentiert, die sich zum Ziel setzen, zur Bewusstmachung der individuellen Mehrsprachigkeit des Lernenden beizutragen und das aufgenommene Sprachmaterial in der Mutter- und Fremdsprache zu systematisieren.

\section{Linguistische und didaktische Grundlagen der Mehrsprachigkeit}

Die Mehrsprachigkeit umfasst zwei Formen, die erste ist die soziale (gesellschaftlich - kollektive) Mehrsprachigkeit, die die Mehrsprachigkeit der Muttersprache, der Region, in der man aufwächst, und die sprachliche Vielfalt der Welt umfasst. Die zweite Form wird als Mehrsprachigkeit des Individuums verstanden (vgl. Wandruszka, 1979: 13-40). Mit Blick auf die Komplexität der individuellen Mehrsprachigkeit unterscheidet Bausch fünf Formen. Es sind: die minimale Mehrsprachigkeit (eine sehr geringe Kompetenz in einer oder mehreren Fremdsprachen), die maximale Mehrsprachigkeit (eine native like Kompetenz in einer oder mehreren Fremdsprachen), die symmetrische Mehrsprachigkeit (Individuen mit einem ungefähr gleichgewichtigen Sprachstand in einer oder mehreren Sprachen), die dominante Mehrsprachigkeit (Individuen mit mehreren Fremdsprachen und einer dominierenden Fremdsprache) und die semilinguale Mehrsprachigkeit (Individuen mit quantitativen oder qualitativen Defiziten in allen ihren Sprachen). In Bezug auf die gesellschaftlich - kollektive Mehrsprachigkeit werden je nach dem soziokulturellen Kontext, der Erwerbssituation oder der Fremdsprachenlernsituation drei Typen der Mehrsprachigkeit unterschieden. Es sind die funktionale Mehrsprachigkeit (mit individuellen Kommunikationsintentionen), die Mehrsprachigkeitsformen, die sich von der Art ihrer Speicherung im Gehirn beschreiben lassen und die Mehrsprachigkeitsformen, die sich von dem Faktor Alter bestimmen lassen (vgl. Bausch, 2003: 439-442).

Auch in der Sprachdidaktik gewinnt die Mehrsprachigkeit immer mehr an Bedeutung. Im Rahmen der fremdsprachendidaktischen Diskussion werden vier 
Mehrsprachigkeitsdidaktikmodelle unterschieden. Das erste bildet die Tertiärsprachendidaktik, bei der die Fremdsprachenreihenfolge die entscheidende Rolle spielt (z.B. das Konzept DaFnE - Deutsch als zweite Fremdsprache nach Englisch), das zweite Modell ist die Mehrsprachigkeitsdidaktik, deren Ziel ist, den Lernenden für Sprachen zu sensibilisieren und sein Sprachbewusstsein, wie auch sein Sprachlernbewusstsein zu fördern, das dritte Modell ist die Interkomprehensionsdidaktik, die eine rezeptiv orientierte mehrsprachige Verstehensstrategie entwickeln lässt, das vierte Modell stellt das Gesamtsprachencurriculum dar (vgl. Hufeisen, 2011: 268-271; Polzin-Haumann und Reissner, 2013: 70-73). Das von Hufeisen erarbeitete Gesamtsprachencurriculum ist ein prototypisches Modell, das einen planerischen Rahmen schafft, in dem VertreterInnen der jeweiligen Sprachen, wie auch die VertreterInnen der jeweiligen Sachfächer mitarbeiten können. Die Verbindung vom Sachfachlernen und mit dem (Fremd-)Sprachenlernen zielt darauf ab, alle Aspekte des Sprachenlernens zu integrieren, um den Synergieeffekt nutzbar zu machen. Die finale Version des Gesamtsprachencurriculums sieht vor, die Sachfächer in der Verkehrssprache und in einer der angebotenen Fremdsprachen zu unterrichten (vgl. Hufeisen, 2011: 271-274).

Im folgenden Beitrag wird auf die individuelle Mehrsprachigkeit fokussiert, deren Bewusstmachung und Förderung im schulischen Erwerb der Mutter- und Fremdsprache/-n stattfinden sollte. Es ist somit von der Mehrsprachigkeit des Fremdsprachenlernenden die Rede, die in Anlehnung an Weinreich mit dem Erlernen der zweiten Fremdsprache beginnt (vgl. Weinreich, 1976: 15-20).

Der Ursprung der Mehrsprachigkeitsdidaktik ist in der Theorie des Bilingualismus von Grosjean zu suchen. Laut Grosjean erfolgt der Erwerb der zweiten und jeder weiteren Sprache interaktiv und nicht additiv: (...) bilingual is not the sum of two complete or incomplete monolinguals; rather, he or she has a unique and specific linguistic configuration. (Grosjean, 1985: 467). De Angelis und Selinker erweitern die Theorie von Grosjean und konstatieren, dass die Sprachen, über die eine mehrsprachige Person verfügt, sich in einer sich stets verändernden Konfiguration befinden, deren Fluktuationen durch mehrere Faktoren beinflusst werden. Zu den Faktoren gehören unter anderen die Kenntnis der jeweiligen Sprache, die Motiviation zum Lernen oder die Möglichkeit zur Anwendung der Sprache (vgl. De Angelis und Selinker, 2001: 4757). Von Cook stammt der Begriff multilinguale Kompetenz. Laut Cook haben die Multilingualen, im Unterschied zu den Monolingualen, eine höhere Sprachbewusstheit und sind überlegen im divergenten Denken: The multicompetenced child has a sharpended metalinguistic awareness of language comparend to the monolingual (Cook, 1993: 4). 
Bevor auf das Konzept der Mehrsprachigkeitsdidaktik Bezug genommen wird, muss erläutert werden, wie die individuelle Mehrsprachigkeit sich herausbildet und wodurch sie bedingt ist. Die Entwicklung der individuellen Mehrsprachigkeit verläuft in mehreren Etappen. Die erste Phase ist der Erwerb der Muttersprache. Im Muttersprachenunterricht erkennt der Lernende die Sprache sowohl in einer ontologischen, als auch in einer epistemologischen Dimension. Die Spezifik der Sprache wird im Kontext der menschlichen Erkenntnis und im Komunikationssystem dargestellt, in dem Sprache als Werkzeug menschlichen Handelns fungiert. Der Muttersprachenunterricht stellt ebenfalls eine Gelegenheit dar, dem Lernenden einen umfassenden Einblick in die Mehrdimensionaliät der Sprache zu gewähren, die grammatischen Kategorien, die Funktion und die Pragmatik der Sprache zu verdeutlichen. In dieser Phase wird dem Lernenden zum ersten Mal bewusst, dass die Region und die Welt mehrsprachig sein können. So können Dialekte und Minderheitensprachen thematisiert und die Sprachen der Nachbarländer präsentiert werden. Schon auf dieser Etappe sind interlinguale Aktivitäten angebracht. Die können dadurch erfolgen, dass im Polnischunterricht die Elemente der kaschubischen Sprache oder der Mundarten (z.B. Schlesisch) miteinbezogen werden. Als entsprechendes didaktisches Material bieten sich Lieder oder Kinderreime an, aber auch kurze Erzählungen und Geschichten. Die ersten Lehrenden, die die individuelle Mehrsprachigkeit fördern, sollten also die Polnischlehrenden sein, und das auf jeder Etappe der Ausbildung, vom Kindergarten über die Grundschule bis zur Oberschule. Mit dem Erlernen der ersten Fremdsprache enthüllt sich für den Lernenden eine neue Dimension der Sprache. Der Lernende macht zum ersten Mal die Erfahrung, Vokabeln und grammatische Strukturen lernen zu müssen und trotzdem nicht alles Gelesene oder Gehörte zu verstehen. Der Erwerb des Wortschatzes und das Erlernen der Grammatik erfolgen über die Brücke der Muttersprache. Diese natürliche Fähigkeit zu vergleichen sollte im Unterricht der ersten Fremdsprache genutzt werden. Dies bedeutet eine Abwendung von dem Prinzip, den Unterricht ausschließlich in der Fremsprache abzuhalten. Es wird vielmehr eine moderne kontrastiv-konfrontative Grammatik angestrebt, deren Ziel ist, die Sprachbewusstheit des Lernenden zu entwickeln. In Bezug darauf schlägt Gozdawa Gołębiowski (2005: 102-105) eine Methode vor, vor der Besprechung der grammatischen Regel in der Fremdsprache über das betroffene grammatische Phänomen in der Muttersprache zu diskutieren und somit die Gesetzmäßigkeiten der Muttersprache bewusst zu machen und auf die fremdsprachliche Regel vorzubereiten. Die Erfahrungen mit dem Erlernen der ersten Fremdsprache führen zur Entwicklung der Fremdsprachenlerntechniken. Der Lernende muss auch lernen lernen. Zumal die Sprache Träger der Kultur ist und 
zugleich Kultur kreiert, eröffnet der Fremdsprachenunterricht die Tür zur Welt und schafft Raum für die Erziehung zur Toleranz gegenüber anderen Sprachen und Kulturen. In Polen hat sich mitlerweile Englisch als erste Fremdsprache etabliert, die weltweit als Sprache der Wissenschaft, der Technik, der Touristik und der Kommunikation gilt. Unter Fremdsprachendidaktikern verbreitet sich jedoch inzwischen die Meinung, dass der Erwerb des Englischen als erster Fremdsprache die individuelle Mehrsprachigkeit des Lernenden beeinträchtigt. Es ist nämlich schwieriger die Motivation zum Erlernen einer zweiten Fremdsprache zu entdecken, wenn man über eine weltweit anerkannte Fremdsprache verfügt. In Bezug darauf wird immer stärker dafür plädiert, nicht mit Englisch, sondern mit einer anderen Fremdsprache zu beginnen (vgl. Krumm, 2005: 29). Das Erlernen der zweiten und jeder nächsten Fremdsprache stellt den Lernenden und den Lehrenden vor neue Aufgaben und neue Herausforderungen. Der Lernende verfügt über gesicherte Kenntnisse in der Muttersprache und hat wenigstens Grundkenntnisse in der ersten Fremdsprache. Er ist auch erfahrener, seine intellektuelle Reife und ein höheres Niveau der bewussten Reflexivität erlauben ihm, Sprachphänomene kritisch zu betrachten und Sprachsysteme zu vergleichen. Auf dieser Etappe werden auch die Fremdsprachenlerntechniken aufgebaut, die beim Erlernen der ersten Fremdsprache entwickelt wurden. Es bildet sich eine spezifische Metakompetenz heraus, die die Grundlage für das Erlernen jeder nächsten Sprache schafft.

\section{Das Konzept der Mehrsprachigkeitsdidaktik}

Den Rahmen für die Mehrsprachigkeitsdidaktik bilden drei Faktoren: das im Vergleich zum Erlernen der ersten Fremdsprache höhere Alter des Lernenden, die Sequenzialisierung des Fremdsprachenerwerbs im schulischen System und die zur Verfügung stehende Zeit zum Erlernen der zweiten und jeder nächsten Fremdsprache. Das höhere Alter des Lernenden sollte sich auf die Auswahl des didaktischen Materials auswirken. Dies bedeutet, dass die zu bearbeitenden Themen der intellektuellen Fähigkeit des Lernenden angepasst werden und eine Möglichkeit anbieten sollten, die sozial- und kulturspezifischen Aspekte tiefer und breiter als bisher aufzugreifen und zu diskutieren und damit dazu beizutragen, die Motivation zum Fremdsprachenlernen zu erhöhen. Das deklarative Wissen (die Polnischkenntnisse und die Erstfremdsprachenkenntnisse) und das prozedurale Wissen des Lernenden (die Fremdsprachenlerntechniken) sollten genutzt werden, um die sprachlichen und die pragmatischen Aspekte der Sprache vergleichen und die Lerntechniken weiter entwickeln zu können. In Bezug auf die Sequenzialisierung des Lernprozesses im schulischen System sollte darauf aufgebaut werden, dass jede involvierte 
Sprache, einschließlich der Muttersprache, als Brücke für das Erlernen der nächstfolgenden Fremdsprache zum Nutzen gereichen sollte. ${ }^{1}$ Die (Fremd-)sprachen, über die der Lernende verfügt, können als Interpretationshilfe bei der Besprechung der landeskundlichen Aspekte dienen. Hervorzuheben ist ebenfalls die Rolle des positiven Transfers, der beim Bearbeiten fremdsprachlicher Texte, bei der Aneignung neuer Vokabeln, wie auch bei der mündlichen und schriftlichen Produktion Unterstützung gewährt. Der negative Transfer kann wiederum als Grundlage dienen, Unterschiede in Sprachsystemen zu veranschaulichen und auf Interferenzfehler aufmerksam zu machen. Die Sequenzialisierung des Fremdsprachenlernprozesses sollte aber auch bedeuten, dass die Fremdsprachenlehrenden und Lehrende für andere Fächer gegenseitig Einblick in die Arbeitsmethoden der Kolleginnen und Kollegen gewinnen, was Grundlagen dafür schaffen kann, fächerübergreifende Projekte zu unternehmen. Die verkürzte Lernzeit sollte die Ökonomisierung des Lehrprozesses zur Folge haben, so dass die Funktionalität der Sprache, die Systematisierung des erworbenen Wissens und die Vertiefung des Könnens explizit hervorgebracht werden. Dies sollte dazu beitragen, die Lehrziele den Bedürfnissen des Lernenden anzupassen und die für ihn relevanten Aspekte der Sprache zu betonen (vgl. Bausch und Kleppin, 1990: 214-216; Polzin-Haumann und Reissner, 2013: 68-72).

\section{Aufgaben für die Polnisch- und Fremdsprachenlehrer/-innen}

Die psychologischen und schulischen Bedingungen, die den Rahmen für die Mehrsprachigkeitsdidaktik schaffen, bilden zugleich eine Grundlage für die Festlegung der Aufgaben für die Polnisch- und Fremdsprachenlehrer/-innen. Die folgende Beschreibung der Aufgaben stützt sich auf die Publikationen von Bausch, Kleppin (1990), Hufeisen (2005), Krumm (2005) und Neuner (2005).

\subsection{Polnischlehrer/-innen}

Die Polnischlehrer/-innen stehen vor der Herausforderung, ihren Unterricht mit speziellem Fokus auf die Vielfalt der Muttersprache und ihrer Varianten zu organisieren. Zu den einzelnen Aufgaben gehören:

- Sensibilsierung auf Sprache und ihre Phänomene, Arbeitsschwerpunkte: Sprachspiele, Kinderreime, Aufzählungen, Lieder, Sprüche,

\footnotetext{
${ }^{1}$ Die vorher gelernte Fremdsprache kann die Unterrichtssprache für die als nächste zu erlernende Fremdsprache sein. Dies ist in Luxemburg der Fall, wenn im Primarbereich in den ersten Stunden Spanisch durch Französisch und Italienisch durch Spanisch eingeführt werden (vgl. Hufeisen, 2005: 11).
} 
Lautspiele, Rhythmusspiele, Experimente mit Sprachmelodie und Tonlagen, Experimente mit der Sprache, Erfindung eines geheimen Kodes für die Unterrichtssprache in der Klasse, Wörterketten etc.;

- Sensibilisierung auf die Varianten der Muttersprache, Erziehung zur Tolerenz und Achtung für alle Varianten des Polnischen, Arbeitsschwerpunkte: Vergleiche zwischen der Umgangssprache und dem Hochpolnischen, zwischen der Sprache zu Hause und der Sprache in der Schule, Arbeit mit Soziolekten, Arbeit mit literarischen Texten etc.;

- Sensibilisierung auf die sprachliche Vielfalt Polens, Erziehung zur Toleranz und Achtung für alle in Polen auftretenden Sprachen (Sprachen der nationalen Minderheiten, Sprachen der ethnischen Minderheiten und Sprachen der regionalen Minderheiten)²; Arbeitsschwerpunkte: Arbeit mit der Landkarte, Entdeckung der Gemeinsamkeiten im Bereich des Wortschatzes, Bearbeitung gewählter Rundfunksendungen und Presseartikel, Erkundung der eigenen Region, Suche nach den Spuren der Mundarten und Minderheitssprachen in der Stadt und in der Region, Besichtigung der Freilichtmuseen etc.;

- Sensibilisierung auf die sprachliche Vielfalt in Europa und in der Welt, Arbeitsschwerpunkte: Arbeit mit der Europakarte und der Weltkarte, Entdeckung der Gemeinsamkeiten im Bereich des Wortschatzes im Rahmen der jeweiligen Sprachfamilie, Bearbeitung gewählter Lieder und kurzer literarischer Formen, Suche nach den Spuren fremder Sprachen in der eigenen Stadt und in der Region etc.;

\footnotetext{
2 Den Status der nationalen Minderheit besitzt die Minderheit, die unter die Bestimmungen des Rahmenübereinkommens zum Schutz nationaler Minderheiten des Europarates fällt. Die nationale Minderheit ist staatsrechtlich anerkannt. In Polen werden neun nationale Minderheiten unterschieden, es sind die Weißrussen, die Tschechen, die Litauer, die Deutschen, die Armenier, die Russen, die Slowaken, die Ukrainer und die Juden. Zu den vier ethnischen Minderheiten zählen die Tataren, die Karaimer, die Sinti und Roma und die Lemken. Die regionale Minderheit im Gebiet Pommern bilden die Kaschuben. Die zahlreichste nationale Minderheit bilden die Deutschen (144.238 Personen mit nur deutscher oder mit deutscher und polnischer Staatsangehörigkeit - Volkszählung 2011), die zweitreichste nationale Minderheit sind die Weißrussen (43.880 Personen mit nur weißrussischer und polnischer Staatsangehörigkeit - Volkszählung 2011)(vgl. http://mniejszosci.narodowe .mac.gov.pl/mne/mniejszosci/charakterystyka-mniejs/6480,Charakterystyka-mniejszosci-narodowych-i-etniczn ych-w-Polsce.html, DW 27.10.2014). Die gesetzlichen Regelungen über die Förderung der nationalen, ethnischen und regionalen Minderheiten in den pädagogischen Einrichtungen in Polen enthält das Gesetzblatt Jahrgang 2014/263.
} 
- die Förderung des prozeduralen Wissens und Bewusstmachung der Lerntechniken, Arbeitsschwerpunkte: Diskussion über die Lerntechniken, Lernen durch Handeln, Lernen durch Diskutieren, Lernen durch Projektarbeit etc.

\subsection{Lehrer/-innen für die erste Fremdsprache}

Die besondere Rolle der Lehrer/-innen der ersten Fremdsprache besteht darin, Grundlagen für das Erlernen der nächsten Fremdsprachen zu schaffen und Motivation zum Weiterlernen zu fördern. Die konkreten Aufgaben sind wie folgt zu beschreiben:

- Förderung der Motivation zum Fremdsprachenlernen, Arbeitsschwerpunkte: Anwendung unterschiedlicher Arbeitstechniken und abwechslungsreicher, authentischer didaktischer Materialien und deren Anpassung an das Alter der Lernenden, ihr Lernprofil und ihre Interessen etc.;

- Berücksichtigung individueller Wahrnehmungs- und Lernpräferenzen der Lernenden, Arbeitsschwerpunkte: Anwendung unterschiedlicher, an den jeweiligen Lernstil angepasster Übungen;

- Anerkennung und Berücksichtigung der individuellen Mehrsprachigkeit der Lernenden, Arbeitsschwerpunkte: Aktivierung der Lernenden mit Migrationshintergrund und deren Eltern, Aktivierung der Lernenden, die eine andere Fremdsprache außerhalb der Schule erwerben, Aktivierung der Lernenden nach längeren Auslandsaufenthalten ${ }^{3}$ etc.;

- Kooperation mit Lehrer/-innen für andere Schulfächer, Arbeitsschwerpunkte: Projektarbeiten wie Kunstunterricht mit Englisch, Sportunterricht mit Englisch, Diskussion über englische Entlehnungen im Polnischen etc.;

- Sensibilisierung auf die Vielfalt der Sprachen und die Vielfalt der Kulturen in der Welt, Erziehung zur Toleranz gegenüber Ausländern, ihrer

\footnotetext{
${ }^{3}$ In den Jahren 2013-2015 wurde in Polen das internationale Projekt Multilingual Families realisiert. Das von der EU finanzierte Projekt fand im Rahmen des Programms Life Long Learning statt und war eine Zusammenarbeit zwischen Partnern aus Polen, Deutschland, Tschechen, Spanien, Großbritannien und Österreich. Das Projekt richtete sich an alle Gruppen der Interessenten, an die Kinder, Eltern, Elternorganisationen, Pädagogen und die pädagogischen Einrichtungen. Die Idee des Projekts beruhte darauf, zur Bewahrung, Belebung und Förderung der kulturellen und sprachlichen Vielfalt Europas beizutragen. Eines der Projektziele war es, pädagogisches Material zu entwickeln und zu sammeln, mit dem der multilinguale Spracherwerb bewusst gemacht und unterstützt werden kann (vgl. http://edukator.koweziu.edu.pl/index.php/ksztalcenie-ustawiczne/1050-projekt-rodziny-wielojezyczne-multilingual-families-jako-narzedz ie-wspierania-nauczycieli-w-nowych-wyzwaniach-wspolczesnego-swiata; https://sites. google.com/site/multilingualfamiliesprojectpl/materialy, DW 22.05.2015).
} 
Sprache und Traditionen, Arbeitsschwerpunkte: Diskussion über soziokulturelle Probleme, Projektarbeiten etc.;

- Entwicklung der Fremdsprachenlerntechniken, Arbeitsschwerpunkte: Diskussion über Techniken zum Wortschatzlernen, zum Erschließen der Bedeutung neuer Vokabeln, zum Lese- und Hörverstehen fremdsprachlicher Texte, zum Optimalisieren des Lernprozesses etc.;

- Förderung der Sprachbewusstheit durch gezielte Vergleiche zur Muttersprache, Arbeitsschwerpunkte: Vergleich der Strukturen der Muttersprache und der Fremdsprache, Bewusstmachung des internationalen Wortschatzes und der Entlehnungen etc.;

- Weiterbildung im Bereich der Mehrsprachigkeitsdidaktik.

\subsection{Lehrer/-innen für die zweite und weitere Fremdsprache(-n)}

Die wichtigste Aufgabe, die den Lehrenden für die zweite und weitere Fremdsprache zugetraut wird, ist die Motivation zum Erlernen verschiedener Fremdsprachen zu erhalten und auf die Vorzüge, die die Kenntnis mehrerer Fremdsprachen mit sich bringt, explizit einzugehen. Der Aufgabenbereich kann folgende Aspekte umfassen:

- Sequenzierung der Sprachfertigkeiten vom Rezeptiven zum Produktiven, Arbeitsschwerpunkte: Diskussion über das Verstehen als Grundlage und Ausgangspunkt für das Sprechen und Schreiben etc.;

- Bewusstmachung der Rolle des positiven Transfers, Arbeitsschwerpunkte: der gemeinsame Wortschatz bei verwandten Sprachen, z.B. Englisch - Deutsch, die Anwendung der Interkomprehenz zur Förderung der rezeptiven Fertigkeiten ${ }^{4}$ etc.;

- Nutzung des deklarativen Wissens der Lernenden, Arbeitsschwerpunkte: Förderung der Sprachbewusstheit durch Vergleiche der Sprachsysteme der Muttersprache und der involvierten Fremdsprachen, Besprechung der Parallelen, Bewusstmachung der Unterschiede, Vorbeugung von Interferenzfehlern, Analyse schriftlicher Arbeiten im Hinblick auf den negativen Transfer etc. (vgl. Hinc, 2014);

- kontinuierliche Förderung des prozeduralen Wissens, Arbeitsschwerpunkte: die Weiterentwicklung der beim Erlernen der ersten Fremdsprache

\footnotetext{
${ }^{4}$ Für die Förderung der rezeptiven Fertigkeiten wurde das Projekt EuroCom entwickelt, das darauf abzielt, Erschließungstechniken zu benutzen, die das Leseverstehen im Rahmen der verwandten Sprachen einer Familie erleichtern. Für die romanischen Sprachen gibt es das Projekt EuroComRom (vgl. Klein und Stegmann, 1999), für die germanischen Sprachen EuroComGerm (vgl. Hufeisen und Marx, 2007), für die slavischen EuroComSlav (vgl. http://www.eurocomslav.de/BIN/inhalt.htm, DW 15.05.2015).
} 
entwickelten Fremdsprachenlerntechniken, Diskussion über die Arbeitstechniken im Unterricht, Vergleich über die Übungstypen aus dem Polnischunterricht, dem Unterricht in der ersten Fremdsprache und in der zweiten Fremdsprache, Anpassung der Übungstypen an die Spezifik und an die Bedürfnisse der Lernergruppe und der einzelnen Lernenden etc. (vgl. Neuner, 2005: 62-63);

- Besprechung der soziokulturellen Aspekte, Arbeitsschwerpunkte: Vergleich der Kulturen über Film, Literatur oder Presseartikel, Diskussion über Stereotypen etc.;

- Kooperation mit Polnischlehrern/-innen und anderen Fremdsprachenlehrern/-innen, Arbeitsschwerpunkte: Vergleich von angewandten Lehrwerken, Arbeitsmethoden und Übungstypen, gemeinsame Projekte, wie zum Beispiel die Übersetzung der Gedichte von Rilke ins Englische und Polnische, Ausflüge in der Region, Vergleich der Informationen für ausländische Touristen (Stadtpläne, Werbungen) etc.;

- Weiterbildung im Bereich der Mehrsprachigkeitsdidaktik, Teilnahme an Seminaren, Konferenzen und Workshops etc ${ }^{5}$.

\section{Abschließende Bemerkungen}

Es unterliegt keinem Zweifel, dass die Verwirklichung der dargestellten Aufgaben curricular verankert werden muss. Damit die Mehrsprachigkeitsdidaktik

\footnotetext{
${ }^{5}$ In den letzten Jahren finden in Polen immer mehr Konferenzen und Seminare statt, es werden Projekte unternommen, die der Problematik der sprachlichen und kulturellen Vielfalt und ihrer Förderung in der Schule gewidmet sind. Als Beispiele kann man folgende Veranstaltungen anführen, die Konferenz „Dzieci wielojęzyczne i wielokulturowe w polskim systemie edukacji”, die im September 2012 in Rawa Mazowiecka im Auftrag des Ministeriums für Bildung und des Ministeriums für die Verwaltung und Digitalisierung abgehalten wurde (vgl. http://www.ore.edu.pl/index.php?option=com_content\&view=article\&id=2744\%3Adzieci-wielojzyczne-i-wielokulturowe-w-polskim-syste mie-edukacji-sprawozdanie-zkonferencji\&catid=106\%3Aspecjalne-potrzeby-edukacyjn e-aktualnoci\&ltemid=1081, DW 20.05.2015), die Konferenz „Wielojęzyczność i wielokulturowość w polskiej szkole”, die 2013 im Zentrum für die Entwicklung der Bildung (Ośrodek Rozwoju Edukacji) in Warschau stattfand (vgl. http://www.ore.edu.pl/index.php?option=com_content\&view=article\&id=3019:konferencja-wielojzyczno-i-wielokulturowo-w-polskiej-szkole\&catid=131:szkolenia\&ltemid=1558, DW 20.05.2015) oder die Konferenz „Dziecko wielojęzyczne i wielokulturowe w polskiej szkole”, die ebenfalls 2013 in dem Lebuser Woiwodschaftsamt stattfand (vgl. http://www.ko-gorzow.edu.pl/ konferencje-i-narady/1883-podsumowanie-konferencji-dziecko-wielojezyczne-i-wielokulturowe-w-polskiej-szkole, DW 20.05.2015).
} 
in Polen und die sich daraus ergebenden Aufgaben für die Lehrer/-innen nicht als rein postulative Ideen betrachtet werden, muss auf vielen Ebenen umgedacht werden. Die erste ist die bildungsministeriale Ebene, auf der Grundlagen für die Bewahrung und Förderung der Mehrsprachigkeit des Lernenden geschaffen werden sollten. Die zweite Ebene betrifft die Lehrkräfte, die stärker als bisher die involvierten Sprachen, einschließlich des Polnischen, in möglichst vielen Dimensionen integrativ heranziehen und besprechen sollten. Darüber hinaus gibt es auch noch andere Organisationen und Institutionen, die für die Entwicklung und Umsetzung der Mehrsprachigkeitsdidaktik eine nicht zu unterschätzende Rolle spielen. Gemeint sind hier die Lehrwerkautoren, die entsprechendes didaktisches Material herausgeben, die Universitäten und andere Bildungseinrichtungen, die Lehrkräfte aus- und weiterbilden. Solange die Hochschulbildung im Rahmen der philologischen Studiengänge darauf abzielt, Fremdsprachenlehrer/-innen und nicht Experten/-innen für die Mehrsprachigkeitsdidaktik auszubilden, kann es diffizil sein, die Postulate der Mehrsprachigkeitsdidaktik in der Unterrichtspraxis anzuwenden.

\section{BIBLIOGRAPHIE}

Bausch, K.-R. 2003. „Zwei- und Mehrsprachigkeit: Überblick“ (w) Handbuch Fremdsprachenunterricht (red. K.-R. Bausch i in.). Tübingen, Basel: Francke: 439-445.

Bausch, K.-R., Kleppin, K. 1990. „Zu den Besonderheiten des Lehrens und Lernens von Deutsch als zweiter Fremdsprache. Thesen und Empfehlungen eines Expertenkolloquiums des Goethe-Instituts". Neusprachliche Mitteilungen aus Wissenschaft und Praxis 43: 213-216.

Cook, V. 1993. „Wholistic Multi-Competence - jeu d'esprit or paradigm shift?” (w) Current Issues in European Second Language Acquisition Research (red. B. Kettemann, W. Wieden). Tübingen: Gunter Narr: 3-9.

De Angelis, G., Selinker, L. 2001. „Interlanguage Transfer and Competing Linguistic Systems in the Multilingual Mind" (w) Cross-linguistic influence in Third Language Acquisition: Psycholinguistic Perspectives (red. J. Cenoz, B. Hufeisen, U. Jessner). Clevedon: Multilingual Matters: 42-58.

Gozdawa-Gołębiowski, R. 2005. „Błąd językowy i gramatyka pedagogiczna”. Kwartalnik Pedagogiczny 1: 95-106.

Grosjean, F. 1985. „The bilingual as a competent but specific speaker-hearer”. Journal of Multilingual and Multicultural Development 6: 467-477.

Hinc, J. 2014. „Zur Förderung der Sprachbewusstheit - Vergleichen und Besprechen der grammatischen Strukturen". Neofilolog 42(1): 39-50.

Hufeisen, B. 2005. „Gesamtsprachencurriculum. Einflussfaktoren und Bedingungsgefüge" (w) Gesamtsprachencurriculum - Integrierte Sprachendidaktik - Common Curriculum. Theoretische Überlegungen und Beispiele der Umsetzung (red. B. Hufeisen, M. Lutjeharms). Tübingen: Gunter Narr: 9-18. 
Hufeisen, B. 2011. „Gesamtsprachencurriculum: Weitere Überlegungen zu einem prototypischen Modell” (w) „Vieles ist sehr ähnlich." - Individuelle und gesellschaftliche Mehrsprachigkeit als bildungspolitische Aufgabe. (red. R. Baur, B. Hufeisen). Baltmannsweiler: Schneider Hohengehren: 265-282.

Hufeisen, B., Marx, N. (red.) 2007. EuroComGerm - Die sieben Siebe: Germanische Sprachen lesen lernen. Aachen: Shaker Verlag.

Klein H. G., Stegmann T. D. (red.). 1999. EuroComRom - Die sieben Siebe: Romanische Sprachen sofort lesen Können. Aachen: Shaker Verlag.

Krumm, H. J. 2005. „Von der additiven zur curricularen Mehrsprachigkeit: über die Notwendigkeit der Einbeziehung von Minderheiten-, Migranten- und Nachbarsprachen" (w) Gesamtsprachencurriculum - Integrierte Sprachendidaktik Common Curriculum. Theoretische Überlegungen und Beispiele der Umsetzung. (red. B. Hufeisen, M. Lutjeharms). Tübingen: Gunter Narr: 27-36.

Neuner, G. 2005. „Gesamtsprachenkonzept, Mehrsprachigkeitsansatz und Tertiärsprachenlernen im europäischen Rahmen" (w) Gesamtsprachencurriculum Integrierte Sprachendidaktik - Common Curriculum. Theoretische Überlegungen und Beispiele der Umsetzung. Tübingen: Gunter Narr: 51-73.

Polzin-Haumann, C., Reissner, Ch. 2013. „Mehrsprachigkeit und Interkomprehension - von der Wissenschaft in die Praxis”. Journal für EuroLinguistik X(10): 67-75.

Wandruszka, M. 1979. Die Mehrsprachigkeit des Menschen. München: Piper.

Weinreich, U. 1977. Sprachen im Kontakt. Ergebnisse und Probleme der Zweitsprachigkeitsforschung. München: C. H. Beck. 